\title{
A Nonparametric Method for Estimating Partial Correlation Coefficient
}

\section{Ebuh GU* and Oyeka ICA}

Department of Statistics, Faculty of Physical Sciences, Nnamdi Azikiwe University, P.M.B. 5025, Awka, Nigeria

\section{Abstract}

This paper proposed a non parametric method for estimating partial correlation coefficient. An illustrative example was provided. Results generated using this method was compared with Siegel's approach and found to be the same but is easier and less tedious to use in practical application.

Keywords: Probabilities; Birth-weight; Correlation coefficient; Kendall tau; Population

\section{Introduction}

When there are three continuous normally distributed populations, one can estimate the simple correlation coefficient between any two samples drawn from these populations as well as any desired partial correlation coefficient between any two samples drawn from any two of the populations while holding at a constant level values of observations drawn from the third population. This estimation is however strictly speaking not possible if the populations are not continuous and normally distributed. In these situations one would have to resort to the use of some nonparametric statistical techniques which do not require these assumptions. A non-parametric method that is often available for this purpose is the Kendall tau correlation coefficient estimation technique [1].

In the Kendall approach the populations of interest may be measurements on as low as the ordinal scale and need not be continuous or normally distributed. To estimate a simple tau correlation coefficient sample observations of equal size drawn from any two observations $\mathrm{X}$ and Y say, are each ranked either from the smallest to the largest or from the largest to the smallest. Tied observations in each sample are as usual assigned to their mean ranks. The ranks assigned to one of the samples, for example those assigned to observations from $\mathrm{X}$ are now arranged in their natural order from 1 through $n$, the common sample size, together with the labels of subjects or respondents with these ranks. The ranks assigned to the observations in the sample drawn from the second population $\mathrm{Y}$ say, are then juxtaposed against the naturally arranged ranks for the correspondent subjects in the sample drawn from the first population $\mathrm{X}$.

Interest is now to determine the degree of agreement between the rankings of observations from $\mathrm{X}$ with those from $\mathrm{Y}$. Since the ranks of observations from $\mathrm{X}$ are already arranged in their natural order, interest is then actually in determining how many pairs of ranks of observations from population $\mathrm{Y}$ are also in their natural order relative to each other.

Now the maximum possible total number of agreements between the ranks assigned to observations form populations $\mathrm{X}$ and $\mathrm{Y}$ which would be obtained if the rankings are in perfect agreement, in this case, when the rankings of observations form Y are also in their natural order as those for observations from population $\mathrm{X}$. This would result in a total maximum possible agreement score of

$$
S_{\max }=\left(\frac{n}{2}\right)=\frac{n(n-1)}{2}
$$

This is the rationale for Kendall's tau correlation coefficient and is the basis for its estimation as

$$
r_{k}=\frac{S_{o b}}{S_{\text {max }}}
$$

Where $S_{o b}$ is the total sum of 1's (or+) and -I's (or-) obtained by comparing members of each pair of ranks assigned to observations from one of the variables $\mathrm{Y}$ say, in relation to each other when these observations are arranged in accordance with the naturally ordered ranks of the observations from the other variable X. Thus Kendall's tau correlation coefficient is estimated as the ratio of the actual observed total agreement score to the total maximum possible score under perfect agreement.

Use of the values obtained with Equation (2) for any two equal sample observations in the fundamental expression for estimating a partial correlation coefficient between two populations X and Y say, when values of a third population $\mathrm{Z}$ say, are held constant which is also the same expression for obtaining partial correlation coefficients based on the simple tau correlation coefficient $[1,2]$.

$$
r_{x y . z}=\frac{r_{x y}-r_{x z} r_{y z}}{\sqrt{\left(1-r_{x z}{ }^{2}\right)\left(1-r_{y z}{ }^{2}\right)}}
$$

Where $r_{x y}, r_{x z}$ and $r_{y z}$ are respectively the tau correlation coefficients between observations from populations $\mathrm{X}$ and $\mathrm{Y}, \mathrm{X}$ and $\mathrm{Z}$, and $\mathrm{Y}$ and $\mathrm{Z}$. The method suggested by Siegel [2] to obtain $r_{x y z}$ is rather cumbersome and tedious to use in practice. Other authors who have worked on parametric and semiparametric methods that are directly or indirectly related to this include Huang [3], Christensen [4], Abdi [5], Pierre [6], Wang [7], Wasserman [8], Zhangsheng and Xihong [9], Prokhorov [10], Kruskal [11].

We here propose to develop following Equations (2) and (3) a rather more formatted, nonparametric statistical method for the estimation of a partial correlation coefficient between two variables $\mathrm{X}$ and $\mathrm{Y}$ say, when a third variable $\mathrm{Z}$ is held constant, where these variables are not necessary continuous or normally distributed.

${ }^{*}$ Corresponding author: Ebuh GU, Department of Statistics, Faculty of Physical Sciences, Nnamdi Azikiwe University, P.M.B. 5025, Awka, Nigeria, E-mail: ablegod007@yahoo.com

Received November 17, 2012; Accepted November 29, 2012; Published December 06, 2012

Citation: Ebuh GU, Oyeka ICA (2012) A Nonparametric Method for Estimating Partial Correlation Coefficient. J Biom Biostat 3:156. doi:10.4172/21556180.1000156

Copyright: ( 2012 Ebuh GU, et al. This is an open-access article distributed under the terms of the Creative Commons Attribution License, which permits unrestricted use, distribution, and reproduction in any medium, provided the original author and source are credited. 


\section{The proposed method}

Now let $\mathrm{x}_{\mathrm{i}}, \mathrm{y}_{\mathrm{i}}$, and $\mathrm{z}_{\mathrm{i}}$ be respectively the $\mathrm{i}^{\text {th }}$ observation in a random sample of size ' $n$ ' drawn from populations $X, Y$ and $Z$, for $i=1,2 \ldots n$. Populations $\mathrm{X}, \mathrm{Y}$ and $\mathrm{Z}$ may be measurements on as low as the ordinal scale. Suppose the ' $\mathrm{n}$ ' observations $\mathrm{x}_{\mathrm{i}}$ drawn from population $\mathrm{X}$ are ranked from the smallest to the largest say. The ' $n$ ' observations $y_{i}$ drawn from population $\mathrm{Y}$ and the ' $\mathrm{n}$ ' observations drawn from population $\mathrm{Z}$ are similarly ranked. Tied observations in each sample are as usual assigned their mean ranks. Furthermore suppose $x_{i}$ is assigned the rank $r_{i x}, y_{i}$ is assigned the rank $r_{i y}$; and $z_{i}$ is assigned the rank $r_{i z}$, for $i=1$, $2, \ldots, \mathrm{n}$. Also suppose for simplicity but without loss of generality that the $z_{i}$ observations have been arranged in their natural order, from the smallest to the largest so that $r_{i z}=i$ for $i=1,2, \ldots, n$ and that the ranks $r_{i x}$ for observations $\mathrm{x}_{\mathrm{i}}$ from population $\mathrm{X}$ and the ranks $\mathrm{r}_{\mathrm{iy}}$ for observations $\mathrm{x}_{\mathrm{iv}}$ from population $\mathrm{Y}$, have each been arranged to correspond with the now naturally occurring ranks ' $i$ ' for their corresponding sister observations $z_{\mathrm{i}}$ from population $\mathrm{Z}$.

Now define

$$
U_{j k ; x . z}=\left\{\begin{array}{l}
1, \text { if } r_{j x}<r_{k x} \\
0, \text { if } r_{j x}=r_{k x} \\
-1, \text { if } r_{j x}>r_{k x}
\end{array}\right.
$$

For $\mathrm{j}=1,2, \ldots, \mathrm{n} ; \mathrm{k}=1,2, \ldots, \mathrm{n} ; \mathrm{j} \neq \mathrm{k}$. provided the rank assigned to the $\mathrm{k}^{\text {th }}$ observation from population X comes after, that succeeds the rank assigned to the $\mathrm{j}^{\text {th }}$ observation from the same population when these observations are arranged in accordance with the natural ordering or ranking of the corresponding sister observations from population $\mathrm{Z}$ $(\mathrm{j} \neq \mathrm{k})$.

$$
\begin{aligned}
& \text { Let } \\
& \stackrel{+}{\pi_{x}}=P\left(U_{j k ; x . Z}=1\right) ; \stackrel{0}{\pi}_{x}=P\left(U_{j k ; x \cdot z}=0\right) ; \pi_{x}=P\left(U_{j k ; x \cdot z}=-1\right)
\end{aligned}
$$

Now let

$$
S_{X}=\sum_{j=1}^{n} \sum_{\substack{k=1 \\ j \neq k}}^{n} U_{j k ; X . Z}
$$

Now from Equations (4) and (5) we have that

$$
E\left(U_{j k ; x . z}\right)=\stackrel{+}{\pi_{x}}-\bar{\pi}_{x} ; \operatorname{Var}\left(U_{j k ; x . z}\right)=\stackrel{+}{\pi_{x}}+\bar{\pi}_{x}-\left(\begin{array}{l}
+ \\
\pi_{x}-\bar{\pi}_{x}
\end{array}\right)^{2}
$$

Similarly

$$
\begin{aligned}
& E\left(S_{x}\right)=\sum_{j=1}^{n} \sum_{k=1}^{n} E\left(U_{j k ; x . z}\right) \quad \text { that is } \\
& E\left(S_{x}\right)=\left(n^{2}-n\right)\left(\stackrel{+}{\pi}_{x}-\bar{\pi}_{x}\right)=n(n-1)\left(\stackrel{+}{\pi}_{x}-\bar{\pi}_{x}\right)
\end{aligned}
$$

Note that $\bar{\pi}_{x}, \pi_{x}$ and $\bar{\pi}_{x}$ are respectively the probabilities that the rank assigned to the $\mathrm{j}^{\text {th }}$ observation from population $\mathrm{X}$ is less than, equal to or greater than the rank assigned to the $\mathrm{K}^{\text {th }}$ observation from the same population if the rank assigned to this $\mathrm{K}^{\text {th }}$ observation succeeds the rank assigned to the $\mathrm{j}^{\text {th }}$ observation from $\mathrm{X}$ when these observations arranged in accordance with the natural ordering of the ranks assigned to the observation from population $\mathrm{Z}$. These probabilities are estimated as

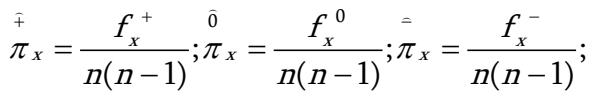

Where $f_{x}^{+}, f_{x}^{0}$ and $f_{x}^{-}$are respectively the number of 1's, 0 's and -1 's in the frequency distribution of these numbers in $U_{j k ; x . z}, j=$ $1,2 \ldots n ; k=1,2 \ldots n ; j \neq k$.

Hence the sample estimate the total number of times the rankings of observations from population $\mathrm{X}$ that are in their natural order and consistent with the natural ordering of the ranks of observations from population $\mathrm{Z}$ less the number of times they are out of order is obtained from equations (8) and (9) as

$$
\left(S_{x}\right)=n(n-1)\left(\stackrel{+}{\pi}_{x}-\bar{\pi}_{x}\right)=f_{x}^{+}-f_{x}^{-}
$$

As noted above, if these rankings are in the natural order then the maximum possible total number of arrangements or score is $S_{\max }=\left(\frac{n}{2}\right)$ (Eqn (1)). Hence the Kendall's tau correlation coefficient between observations in population $\mathrm{X}$ and observations from population $\mathrm{Z}$ may be estimated using Equations (1) and (10) in Equation (2) as:

$$
\begin{aligned}
& r_{k, x z}=r_{x z}=\frac{S_{x}}{S_{\max }}=\frac{S_{x}}{n(n-1) / 2} \\
& \text { or } \\
& r_{x z}=2\left(\begin{array}{l}
\overline{+} \\
\pi_{x}-\pi_{x}
\end{array}\right)=\frac{2\left(f_{x}^{+}-f_{x}^{-}\right)}{n(n-1)}
\end{aligned}
$$

To estimate the correlation coefficient between observations from population $\mathrm{Y}$ and observations from population $\mathrm{Z}$ we define.

$$
U_{j k ; y . z}=\left\{\begin{array}{l}
1, \text { if } r_{j y}<r_{k y} \\
0, \text { if } r_{j y}=r_{k y} \\
-1, \text { if } r_{j y}>r_{k y}
\end{array}\right.
$$

For $\mathrm{j}=1,2 \ldots \mathrm{n} ; \mathrm{k}=1,2 \ldots \mathrm{n} ; \mathrm{j} \neq \mathrm{k}$. provided the rank assigned to the $\mathrm{k}^{\text {th }}$ observation from population $\mathrm{Y}$ comes after, that is succeeds the rank assigned to the $j^{\text {th }}$ observation from the same population when these observations are arranged in accordance with the natural ordering of ranking of the corresponding sister observations from population $\mathrm{Z}(\mathrm{j} \neq \mathrm{k})$

$$
\begin{aligned}
& \text { Let } \\
& \stackrel{+}{\pi_{y}}=P\left(U_{j k ; y . z}=1\right) ; \pi_{y}=P\left(U_{j k ; y \cdot z}=0\right) ; \bar{\pi}_{y}=P\left(U_{j k ; y . z}=-1\right)
\end{aligned}
$$

Also let

$$
S_{y}=\sum_{j=1}^{n} \sum_{\substack{k=1 \\ j \neq k}}^{n} U_{j k ; y . z}
$$

Now from Equations (12) and (13) we have that

$$
E\left(U_{j k ; y, z}\right)=\stackrel{+}{\pi}_{y}-\bar{\pi}_{y} ; \operatorname{Var}\left(U_{j k ; y, z}\right)=\stackrel{+}{\pi}_{y}+\bar{\pi}_{y}-\left({ }^{+} \bar{\pi}_{y}-\bar{\pi}_{y}\right)^{2}
$$

Similarly from Equations (14) and (15)

$$
E\left(S_{y}\right)=n(n-1)\left(\stackrel{+}{\pi}_{y}-\bar{\pi}_{y}\right)
$$


Note that ${ }_{\pi_{y}}^{+},{ }_{\pi_{y}}^{0}$ and $\bar{\pi}_{y}$ are respectively the probabilities that the rank assigned to the $j^{\text {th }}$ observation from population $\mathrm{Y}$ which is less than, equal to or greater than the rank assigned to the $\mathrm{k}^{\text {th }}$ observation from the same population. If the rank assigned to this $\mathrm{k}^{\text {th }}$ observation succeeds the rank assigned to the $\mathrm{j}^{\text {th }}$ observation from $\mathrm{Y}$ when these observations are arranged in accordance with the natural ordering of the ranks assigned to the observations from population $\mathrm{Z}$. These probabilities are estimated as

$$
{\stackrel{\uparrow}{\pi_{y}}}=\frac{f_{y}^{+}}{n(n-1)} ; \pi_{y}=\frac{f_{y}{ }^{0}}{n(n-1)} ; \pi_{y}=\frac{f_{y}^{-}}{n(n-1)} ;
$$

Where $f_{y}^{+}, f_{y}^{0}$ and $f_{y}^{-}$are respectively the number of 1 's, 0 's and -1's in the frequency distribution of these numbers in $U_{j k ; y . z}$ $\mathrm{j}=1,2 \ldots \mathrm{n} ; \mathrm{k}=1,2 \ldots \mathrm{n} ; \mathrm{j} \neq \mathrm{k}$

Hence the sample estimate of the total number of times the rankings of observations from population $\mathrm{Y}$ are in their natural order and consistent with the natural ordering of the ranks of observations from population $\mathrm{Z}$ less the number of times they are out of order is obtained from Equations (16) and (17) as

$$
\left(S_{y}\right)=n(n-1)\left(\stackrel{+}{\pi}_{y}-\bar{\pi}_{y}\right)=f_{y}^{+}-f_{y}^{-}
$$

Again if the ranks are in their natural order then the maximum possible total number of arrangement of scores $S_{\max }=\left(\frac{n}{2}\right)=\frac{n(n-1)}{2}$ given in Equation (1). Hence the Kendall's tau correlation coefficient between observations from population $\mathrm{Y}$ and observations from population $\mathrm{Z}$ may be estimated using Equation using Equations (1) and (18) in Equation (2) as:

$$
r_{y z}=\frac{S_{y}}{S_{\max }}=2\left(\begin{array}{l}
\mp \\
\pi_{y}-\pi_{y}
\end{array}\right)=\frac{2\left(f_{y}^{+}-f_{y}^{-}\right)}{n(n-1)}
$$

Finally to estimate the tau correlation coefficient between observations from population $\mathrm{X}$ and observations from population $\mathrm{Y}$ we may first arrange the ranks of observations from one of the populations X say in their natural order and then tag along the ranks of the corresponding observations from population $\mathrm{Y}$.

$$
U_{j k ; x y \cdot z}=\left\{\begin{array}{l}
1, \text { if } r_{j y}<r_{k y} \\
0, \text { if } r_{j y}=r_{k y} \\
-1, \text { if } r_{j y}>r_{k y}
\end{array}\right.
$$

For $\mathrm{j}=1,2 \ldots \mathrm{n} ; \mathrm{k}=1,2 \ldots \mathrm{n} ; \mathrm{j} \neq \mathrm{k}$

Provided the rank assigned to the $\mathrm{k}^{\text {th }}$ observation from population $\mathrm{Y}$ comes after, that succeeds the rank assigned to the $\mathrm{j}^{\text {th }}$ observation from the same population when these observations are arranged in accordance with the natural ordering or ranking of the corresponding sister observations from population $\mathrm{X}(\mathrm{j} \neq \mathrm{k})$

Also let

$$
\stackrel{+}{\pi}_{x y}=P\left(U_{j k ; x y}=1\right) ; \pi_{x y}=P\left(U_{j k ; x y}=0\right) ; \bar{\pi}_{x y}=P\left(U_{j k ; x y}=-1\right)
$$

\section{Define}

$$
S_{x y}=\sum_{j=1}^{n} \sum_{\substack{k=1 \\ j \neq k}}^{n} U_{j k ; x y}
$$

From equations (20) and (21) we have that

$$
E\left(U_{j k ; x y}\right)=\stackrel{+}{\pi}_{x y}-\bar{\pi}_{x y} ; \operatorname{Var}\left(U_{j k ; x y}\right)=\stackrel{+}{\pi}_{x y}+-_{\pi}^{-}-\left(+_{x y}^{+}-\bar{\pi}_{x y}\right)^{2}
$$

Similarly,

$$
E\left(S_{x y}\right)=n(n-1)\left(\stackrel{+}{\pi}_{x y}-\bar{\pi}_{x y}\right)
$$

Note that $\bar{\pi}_{x y}, \pi_{x y}$ and $\bar{\pi}_{x y}$ are respectively the probabilities that the ranks assigned to the $j^{\text {th }}$ observation in population $\mathrm{Y}$ is less than, equal to or greater than the rank assigned to the $\mathrm{k}^{\text {th }}$ observation in the same population $(\mathrm{Y})$ when these observations have been arranged so that the ranks assigned them correspond with the natural order of the ranks of their sister observations in population $\mathrm{X}$ and are estimated as:

$$
{\stackrel{\mp}{\pi_{x y}}}_{x}=\frac{f_{x y}{ }^{+}}{n(n-1)} ; \pi_{x y}=\frac{f_{x y}{ }^{0}}{n(n-1)} ; \pi_{x y}=\frac{f_{x y}{ }^{-}}{n(n-1)} ;
$$

Where $f_{x y}{ }^{+}, f_{x y}{ }^{0}$ and $f_{x y}{ }^{-}$are respectively the number of 1 's, 0 's and -1 's in the frequency distribution of these numbers in $U_{j k ; x y}$ $\mathrm{j}=1,2 \ldots \mathrm{n} ; \mathrm{k}=1,2 \ldots \mathrm{n} ; \mathrm{j} \neq \mathrm{k}$

Hence the sample estimate of the total number of times the rankings of observations from population $\mathrm{Y}$ are in their natural order and consistent with the natural ordering of their sister observations from population $\mathrm{X}$ less the number of times they are out of order is obtained from equation 24 as:

$$
\left(S_{x y}\right)=n(n-1)\left(\stackrel{+}{\pi}_{x y}-\bar{\pi}_{x y}\right)=f_{x y}^{+}-f_{x y}^{-}
$$

As before if these rankings are in their natural order then the maximum possible total number of arrangements or score is $\mathrm{S}_{\max }$ given in Equation (1). Hence the tau correlation coefficient between observations in populations $\mathrm{X}$ and $\mathrm{Y}$ is estimated by using Equation (1) and (26) in Equation (2) yielding:

$$
r_{x y}=\frac{S_{y}}{S_{\max }}=2\left(\begin{array}{l}
\hat{+}_{x y}-\pi_{x y} \\
\pi^{\prime}
\end{array}\right)=\frac{2\left(f_{x y}{ }^{+}-f_{x y}{ }^{-}\right)}{n(n-1)}
$$

Finally using Equations (11) (19) and (27) in Equation (3) we obtain the proposed non-parametric estimate of the partial correlation coefficient between populations $\mathrm{X}$ and $\mathrm{Y}$ when observations from population $\mathrm{Z}$ are held at a constant level as

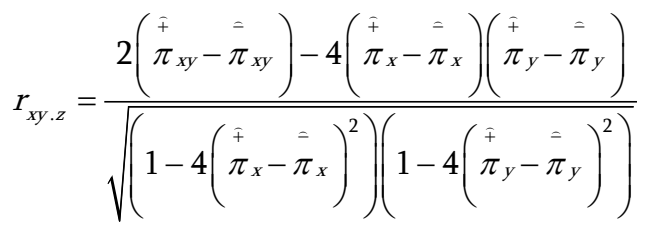

or in terms of the corresponding frequencies in Equations (11) (19) and (27)

$$
r_{x y . z}=\frac{2\left(\left(n(n-1)\left(f_{x y}^{+}-f_{x y}^{-}\right)-2\left(f_{x}^{+}-f_{x}^{-}\right)\left(f_{y}^{+}-f_{y}^{-}\right)\right)\right.}{\sqrt{\left((n(n-1))^{2}-4\left(f_{x}^{+}-f_{x}^{-}\right)^{2}\right)\left((n(n-1))^{2}-4\left(f_{x}^{+}-f_{x}^{-}\right)^{2}\right)}}
$$

\section{Illustrative example}

We here use the proposed method to estimate the partial 
Citation: Ebuh GU, Oyeka ICA (2012) A Nonparametric Method for Estimating Partial Correlation Coefficient. J Biom Biostat 3:156. doi:10.4172/21556180.1000156

Page 4 of 5

correlation coefficient between mother's weight and the birth weight of her most recent birth both in $\mathrm{kg}$ given maternal age in years using the following data on a random sample of 12 mothers.

\begin{tabular}{|l|l|l|l|l|l|l|l|l|l|l|l|l|}
\hline S/N & 1 & 2 & 3 & 4 & 5 & 6 & 7 & 8 & 9 & 10 & 11 & 12 \\
\hline Maternal Age (Z) & 31 & 26 & 30 & 28 & 24 & 36 & 20 & 34 & 25 & 25 & 30 & 24 \\
\hline Maternal Weight (x) & 75 & 63 & 55 & 90 & 60 & 76 & 59 & 73 & 62 & 83 & 75 & 68 \\
\hline Child birth Weight (y) & 3.5 & 3.9 & 3.6 & 2.8 & 3.0 & 3.8 & 2.7 & 3.7 & 2.8 & 2.9 & 3.0 & 3.2 \\
\hline
\end{tabular}

To use the proposed method we here rank maternal age $\left(z_{\mathrm{i}}\right)$ maternal weight $\left(\mathrm{x}_{\mathrm{i}}\right)$ and child birth weight $\left(\mathrm{y}_{\mathrm{i}}\right)$ each from the smallest to the largest assigning the rank ' 1 ' to the smallest, through the rank 12 to the largest observation in each sample. Tied observations or values in each sample are as usual assigned their mean ranks. We here also but without loss of generality arrange the ranks assigned to material age $(z)$ in their natural order. The ranks assigned to the corresponding maternal weight $(\mathrm{z})$ and child birth weight $(\mathrm{y})$ are now tagged along the naturally ordered ranks of their sister observations for material age (z). The results are presented in table 1 .

To estimate the tau correlation coefficient $r_{x z}$, between maternal age $(\mathrm{z})$ and maternal weight $(\mathrm{x})$, we first obtain the value of $\mathrm{u}_{\mathrm{j}, \mathrm{k} ; \mathrm{x}, \mathrm{z}}$ of Equation (4). These values are more easily obtained in the form of a 12 $\mathrm{X} 12$ contingency table (Table 2).

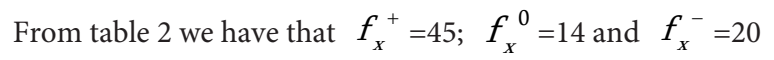

Hence from Equation (9) we have that:

$$
\bar{\tau}_{x}=\frac{45}{12(12-1)}=\frac{45}{132}=0.341 \text { and } \bar{\pi}_{x}=\frac{20}{132}=0.152
$$

Therefore from Equation (11) we have that estimated tau correlation coefficient between maternal age $(\mathrm{z})$ and maternal weight ( $\mathrm{x})$ is:

$$
\mathrm{r}_{\mathrm{xz}}=2(2.341-0.152)=0.378 \text { or } 37.8 \text { percent } \text {. }
$$

To obtain the tau correlation coefficient between maternal age $(\mathrm{z})$

\begin{tabular}{|l|l|l|l|l|l|l|l|l|l|l|l|l|}
\hline Subject No & 7 & 5 & 12 & 9 & 10 & 2 & 4 & 3 & 11 & 1 & 8 & 6 \\
\hline
\end{tabular}

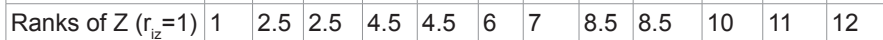
\begin{tabular}{|l|l|l|l|l|l|l|l|l|l|l|l|l|}
\hline Rank of $x\left(r_{i x}\right)$ & 2 & 3 & 6 & 4 & 11 & 5 & 12 & 1 & 8.5 & 8.5 & 7 & 10 \\
\hline
\end{tabular} \begin{tabular}{|l|l|l|l|l|l|l|l|l|l|l|l|l|}
\hline Rank of $y\left(r_{i y}\right)$ & 1 & 5.5 & 7 & 2.5 & 4 & 12 & 2.5 & 9 & 5.5 & 8 & 10 & 11 \\
\hline
\end{tabular}

Table 1: Natural Order of the Ranks of Maternal Age (z) with the Corresponding

\begin{tabular}{|c|c|c|c|c|c|c|c|c|c|c|c|c|c|c|}
\hline & \multicolumn{13}{|c|}{$r_{k x}$} \\
\hline & & t No. & 7 & 5 & 12 & 9 & 10 & 2 & 4 & 3 & 11 & 1 & 8 & 6 \\
\hline & & 2 & 3 & 6 & 4 & 11 & 5 & 12 & 1 & 8.5 & 8.5 & 7 & 10 & \\
\hline \multirow{12}{*}{$r_{j x}$} & 7 & 2 & 0 & 1 & 1 & 1 & 1 & 1 & 1 & -1 & 1 & 1 & 1 & 1 \\
\hline & 5 & 3 & & 0 & 1 & 1 & 1 & 1 & 1 & -1 & 1 & 1 & 1 & 1 \\
\hline & 12 & 6 & & & 0 & -1 & 1 & -1 & 1 & -1 & 1 & 1 & 1 & 1 \\
\hline & 9 & 4 & & & & 0 & 1 & 1 & 1 & -1 & 1 & 1 & 1 & 1 \\
\hline & 10 & 11 & & & & & 0 & -1 & 1 & -1 & -1 & -1 & -1 & -1 \\
\hline & 2 & 5 & & & & & & 0 & 1 & -1 & 1 & 1 & 1 & 1 \\
\hline & 4 & 12 & & & & & & & 0 & -1 & -1 & -1 & -1 & -1 \\
\hline & 3 & 1 & & & & & & & & 0 & 1 & 1 & 1 & 1 \\
\hline & 11 & 8.5 & & & & & & & & & 0 & 0 & -1 & 1 \\
\hline & 1 & 8.5 & & & & & & & & & & 0 & -1 & 1 \\
\hline & 8 & 7 & & & & & & & & & & & 0 & 1 \\
\hline & 6 & 10 & & & & & & & & & & & & 0 \\
\hline
\end{tabular}
Ranks for Maternal Weight $(\mathrm{x})$ and Child Birth Weight (y).

\begin{tabular}{|l|l|l|l|l|l|l|l|l|l|l|l|l|l|}
\hline \multicolumn{2}{|c|}{} & $\mathrm{r}_{\mathrm{ky}}$ \\
\hline Subject No. & 7 & 5 & 12 & 9 & 10 & 2 & 4 & 3 & 11 & 1 & 8 & 6 \\
\hline & 1 & 5.5 & 7 & 2.5 & 4 & 12 & 2.5 & 9 & 5.5 & 8 & 10 & 11 & \\
\hline 7 & 1 & 0 & 1 & 1 & 1 & 1 & 1 & 1 & 1 & 1 & 1 & 1 & 1 \\
\hline 5 & 5.5 & & 0 & 1 & -1 & -1 & 1 & -1 & 1 & 0 & 1 & 1 & 1 \\
\hline 12 & 7 & & & -1 & -1 & 1 & -1 & 1 & -1 & 1 & 1 & 1 \\
\hline 9 & 2.5 & & & & 0 & -1 & 1 & 0 & 1 & 1 & 1 & 1 & 1 \\
\hline 10 & 4 & & & & & 0 & 1 & -1 & 1 & 1 & 1 & 1 & 1 \\
\hline 2 & 12 & & & & & & 0 & -1 & -1 & -1 & -1 & -1 & -1 \\
\hline 4 & 2.5 & & & & & & & 0 & 1 & 1 & 1 & 1 & 1 \\
\hline 3 & 9 & & & & & & & 0 & -1 & -1 & 1 & 1 \\
\hline 11 & 5.5 & & & & & & & & 0 & 1 & 1 & 1 \\
\hline 1 & 8 & & & & & & & & 0 & 1 & 1 \\
\hline 8 & 10 & & & & & & & & & & 0 & 1 \\
\hline 6 & 11 & & & & & & & & & \\
\hline
\end{tabular}

\begin{tabular}{|l|l|l|l|l|l|l|l|l|l|l|l|l|}
\hline Subject No. & 3 & 7 & 5 & 9 & 2 & 12 & 8 & 11 & 1 & 6 & 10 & 4 \\
\hline $\begin{array}{l}\text { Rank for Maternal } \\
\text { Weight }\left(r_{\text {ix }}=1\right)\end{array}$ & 1 & 2 & 3 & 4 & 5 & 6 & 7 & 8 & 9 & 10 & 11 & 12 \\
\hline $\begin{array}{l}\text { Rank for Child Birth } \\
\text { Weight }\left(r_{\text {iv }}\right)\end{array}$ & 9 & 1 & 5.5 & 2.5 & 12 & 7 & 10 & 5.5 & 8 & 11 & 4 & 2.5 \\
\hline
\end{tabular}

Table 4: Natural order of the ranks for Maternal Weight along with the Corresponding Ranks for Child Birth Weight.

and child birth weight (y) we may again calculate the values of $u_{j k y . z}$ (Equation (12) in a tabular form (Table 3).

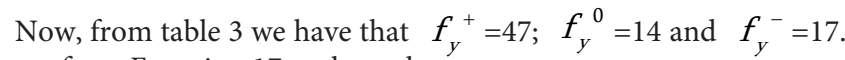
Hence, from Equation 17 we have that:

$$
\stackrel{\mp}{\pi}_{y}=\frac{47}{12(11)}=\frac{47}{132}=0.356 \text { and } \bar{\pi}_{y}=\frac{17}{132}=0.129
$$

Therefore an estimate of the tau correlation coefficient between maternal age ( $\mathrm{z}$ ) and child birth weight (y) is from Equation (19).

$$
\mathrm{r}_{\mathrm{yz}}=2(0.356-0.129)=0.454 \text { or } 45.4 \%
$$

To obtain the tau correlation coefficient between maternal weight (x) and child birth weight (y) we may first arrange the ranks for maternal weight (x) say, in their natural order and then tag along the ranks of the corresponding child birth weight (Table 4).

The calculation of the values of $\mathrm{U}_{\mathrm{jk} \text { :Xy }}$ (Equation (20) is also shown in a tabular form (Table 5).

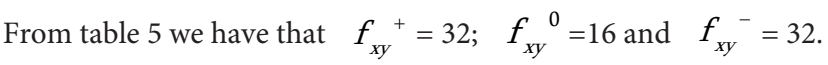

Therefore from Equation (25) we have that,

$$
\stackrel{\mp}{\pi y}_{x y}=\frac{32}{132}=0.242 \text { and } \bar{\pi}_{x y}=\frac{32}{132}=0.242
$$

Hence the estimated tau correlation coefficient between maternal weight and child birth weight is from Equation (27)

$r_{x y}=2(0.242-0.242)=0.000$. Thus, here there is virtually no direct association between maternal weight and child birth weight. The partial correlation coefficient between maternal weight and child birth weight, holding maternal age constant is estimated using these results in Equation (28),

$$
r_{x y . z}=\frac{0-(0.378)(0.454)}{\sqrt{\left(1-(0.378)^{2}\right)\left(1-(0.454)^{2}\right)}}
$$


Citation: Ebuh GU, Oyeka ICA (2012) A Nonparametric Method for Estimating Partial Correlation Coefficient. J Biom Biostat 3:156. doi:10.4172/21556180.1000156

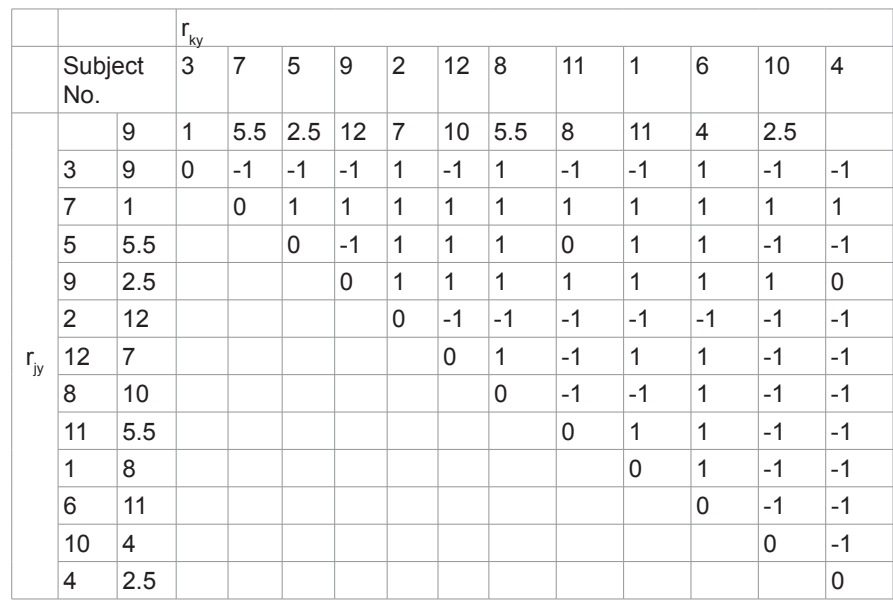

Table 5: Values of ujk;xy for the Data of Table 4.

$$
=\frac{-0.172}{\sqrt{(0.857)(0.794)}}=\frac{-0.172}{0.825}=-0.208
$$

this indicates a negative partial association

This result is the same as the one obtained using Siegel's approach. However the present proposed method seems easier and less tedious to use in practical application.

\section{Summary and Conclusion}

We have in this paper proposed a non parametric method for estimating partial correlation coefficient. This method was used to generate results. Equally, Siegel's approach was used to generate results as well. It was observed that the proposed method generate the same result with Siegel's approach but seems easier and less tedious to use in practical application.

\section{References}

1. Kendall M (1948) Rank Correlation Methods. Charles Griffin and Company Limited.

2. Siegel Sidney (1956) Nonparametric Statistics for the Behavioral Sciences McGraw-Hill Series in Psychology, New York.

3. Huang TM (2010) Testing Conditional Independence Using Maximal Nonlinear Conditional Correlation. Ann Stat 38: 2047-2091.

4. Christensen D (2005) Fast Algorithms for the Calculation of Kendall's T. Comput Stat 20: 51-62.

5. Abdi H (2007) Kendall Rank Correlation in Salkind NJ. Encyclopedia of Measurement and Statistics, Thousand Oaks (CA), Sage.

6. Legendre P (2000) Comparison of Permutation Methods for the Partia Correlation and Partial Mantel Tests. J Stat Comput Simul 67: 37-73.

7. Wang N (2003) Marginal Nonparametric Kernel Regression Accounting for Within Subject Correlation. Biometrika 90: 43-52.

8. Wasserman L (2006) All of Nonparametric Statistics. Springer, New York.

9. Yu Z, Lin X (2010) Semiparametric Regression with Time-Dependent Coefficients for Failure Time Data Analysis. Stat Sin 20: 853-869.

10. Prokhorov AV (2001) Kendall Coefficient of Rank Correlation in Hazewinkel Michiel. Encyclopedia of Mathematics, Springer.

11. Kruskal WH (1958) Ordinal Measures of Association. Journal of the American Statistical Association 53: 814-861. 